\title{
Embryologist's Job in Assisted Reproductive Technologies and in Vitro Fertilization
}

\author{
Harsimrat Kaur ${ }^{1,}$ Ram Dayal ${ }^{*}$, Kamla Singh ${ }^{3}$ \\ ${ }^{1}$ Embryologist (Intern), Institute of Reproduction and Child Cares, Panckhula, Haryana, India \\ ${ }^{2}$ Director and Scientist Sr. Embryologist, IRCC IVF Centre, Panchkula, Haryana, India \\ ${ }^{3} \mathrm{CMD}$ and Sr. Infertility Consultant, Institute of Reproduction and Child Cares, India \\ *Corresponding author's Email: ramdayal2509@gmail.com
}

Article Info
Volume 8, Issue 5
Page Number : 108-114
Publication Issue
September-October-2021
Article History
Accepted : 05 Sep 2021
Published : 15 Sep 2021
*Corresponding Author
Ram Dayal2*, Ph.D.
Director and Scientist Sr.
Embryologist, IRCC IVF
Centre,

Haryana, INDIA.

\section{ABSTRACT}

What exactly does an embryologist do? is one of the most common question asked by patients and the possible answer could be that embryologist is the child's first watchperson. The ability to grow embryos in laboratory environment was a huge scientific achievement. Scientists and Embryologists are involved in reproductive research and fertility treatment. The embryologist has a huge role to play in IVF/ICSI process and the contribution of embryologist is no less than infertility consultant. They might not be doctors, but they are highly trained medical professionals, holding a master's degree or Ph.D. due to specialized nature of work. They are responsible for management and maintenance of laboratory used in creating embryos as well as monitoring those embryos. The important activities that embryologist does are maintaining the embryology lab (temperature, humidity, $\mathrm{CO} 2$ cylinder, diffusion gas and $\mathrm{pH}$ ), oocyte screening during ovum pickup (OPU), incubation and checking of fertilization, embryo transfer, vitrification and embryo biopsy for preimplantation genetic screening (PGS) or pre-implantation genetic diagnosis (PGD).

Keywords : Embryologist, Embryology Lab, Gamete, Embryo Culture, Embryo Transfer, Incubator, Culture Media, Consumables, Air Quality \& sterility, OPU and PGS/PGD.

\section{INTRODUCTION}

The branch of medical science that deals with the formation, growth, and development of embryo is called embryology. It deals with the prenatal stage of in-vitro development from screening and washing of gametes, co-incubation leads to fertilization, formation of zygote, development of embryo till cryopreservation or embryo transfer (ET) and in-vivo implantation and development of foetus to the birth of a new individual [1]. The role of Embryologists is usually underrated and their presence behind the 
journey goes unnoticed. Despite this ignorance, we must always not overlook the very fact that the embryologists are essentially scientists, who play an important role within the successful conception of babies through advanced assisted reproductive technologies. An embryologist helps creating viable embryos to be either used in IVF treatment or while embryo freezing, that is later used for frozen embryo transfer (FET). The responsibilities of embryologist involve maintaining the genetic material that creates embryos and to also take care of the development of the embryos closely. This needs deep understanding of the science behind nurturing eggs, sperm and embryos outside human body and technological backup to ensure success rates. The role of embryologist has also been intensified over the years with the rise of infertility globally - approximately 1 in 7 of reproductive age being diagnosed as infertile. Globally, in a year over five million babies are born through ART treatment. In India, the IVF industry is expected to grow at a compound annual growth rate (CAGR) of $28 \%$, and is expected to be around Rs 495 crore (USD 775 million) by 2022 [2].

\section{NEED OF EMBRYOLOGIST}

Before learning about embryologist, it's crucial to grasp the entire IVF process. It starts from extracting the eggs, retrieving sperm sample, then fertilizing them by conventional IVF or manually by injecting sperm into an egg cytoplasm in the laboratory by using micromanipulator. Further, embryologist must observe the fertilization and grading of zygote (Z1 to Z4, Scott et al, 2003) $[3,18]$, cleavage pattern and degree of fragmentation in-vitro culture conditions. The quality embryos are then transferred into the uterine cavity either on day 3 or day 5. A team of highly trained embryologists perform these procedures in a sophisticated and well-controlled environment laboratory. They're the scientists who help to create embryos outside the womb using sperms and eggs. As more and more couples are now going for IVF once they are unable to conceive naturally, it shows there is a rise within the demand for ART treatments. This also indicates that there will be a requirement for trained embryologists. The more of embryologist demand also will need the necessity of intense knowledge in application of emerging technologies in the near future. An embryologist may be employed by assisted reproductive clinics in government as well as in private hospitals. Gradually, one may also be inclining to knowledgeable positions as Lab manager or Lab director. But embryologist is such employment which needs rigorous training in laboratory skills, management and be updated about the latest technologies. Many educational institutions have now introduced embryology courses in their modules. One may also join these institutes after completion of their course as faculty of embryologist [2].

\section{QUALIFICATION OF EMBRYOLOGIST}

Clinical Embryology is a study of fertilization of eggs and healthy development of embryos. To gain access in field of embryology one has to complete bachelor's degree in life science followed by post graduate qualification, preferably in Assisted Reproductive Technology, life sciences, Clinical Embryology, Reproductive Biology /Biotechnology, Anatomy, Physiology, Microbiology, Veterinary Sciences etc. In India, different universities and institutes offer post graduate degree or post graduate diploma by conducting entrance examinations.

The entrance tests conducted for admission into a clinical embryology courses are AIIMS (All India Institute of Medical Science) and MET (All India Manipal Entrance Test). Students proceeding Clinical Embryology or practicing it are offered prominent jobs by both government and private sectors. In India, there is no government authorized certification but some private organization conducting entrance examination for certification like as: Indian Fertility Society (IFS) [4] and some countries also conducting 
the certification exams for certified clinical embryologist like as: European Society of Human Reproduction and Embryology (ESHRE) [5] and American Society for Reproductive Medicine (ASRM) [6]. In United Kingdom, to enter the field of embryology, one must need a degree in a relevant subject such as biomedical sciences, biology, microbiology, genetics or biochemistry. The candidate can then apply for a place on the National school of Healthcare (NHS) Scientist Training Programme (STP) to train as a clinical embryologist / scientist, specializing in reproductive science. Admission on to the STP is highly competitive and candidates must need a first or 2:1 degree, or a 2:2 with a relevant Masters or $\mathrm{PhD}$, to be considered [7]. In order to carve a successful career in this field, candidates must possess certain skills. These skills are not a part of eligibility criteria, they are still highly desirable [8]. Some of them are listed [9] below:
1) Administrative skills- Embryologists are required to possess great abilities in tackling the in-office environment while keeping a tab on minor details and records.

2) Interpersonal skills- an embryologist is required to collaborate with doctors, physicians, peer groups, families and people from different departments.

3) Management skills- A clinical Embryologist is required to deal with multiple tasks, and hence the job roles are complex.

4) Expertise and experience- A clinical embryologist must possess a great experience in treating patients with diverse complications.

5) Technical knowledge- A clinical embryologist must be well-versed with the technical technologies as well.

\section{ROLE \& SKILLS OF EMBRYOLOGIST}

1 Good knowledge of mammalian 17 embryology \& endocrinology.

2 Awareness of gas cylinders, gas regulator 18 and supply of gas into incubator.

3 Awareness of incubators operation and 19 maintenance.

4 Humidity and temperature management 20 skills.

5 Clinical embryologist must be aware 21 about cleaning protocol, sterility of andrology and embryology lab.

6 Updating time to time theoretical and 22 hands on embryology lab skills.

7 Strong leadership skills needs to 23 interpersonal communication and teaching to team of IVF.

8 Awareness about all required consents 24 forms filling.
Excellent hands on skills of oocyte denudation after conventional IVF and time management.

Excellent hands on skills of oocyte denudation with hyaluronidase enzyme and oocyte grading before ICSI procedure.

Excellent hands on skills in ICSI procedure and careful about damaging of oocyte lead to necrotic.

Embryo development monitoring skills.

Excellent hands on skills of embryo loading in the ET catheter \& transfer into uterine cavity and assuring no embryo returned.

Good knowledge of embryo grading \& selection for transfer to patient uterine cavity.

Excellent hands on skills of oocyte, embryo and blastocyst vitrification.

Excellent hands on skills of embryo thawing for FET. 
9 Keeping accurate and detailed patient 25 records in record file.

10 Ability to determine patient's fertility 26 level and having solution to offer.

11 Speak to patients about specific fertility treatment options.

12 Research and discuss fertility treatment 28 options with other medical staffs.

13 Use of assisted reproductive technologies (ART) to help with infertility couples.

14 Embryologist must have good knowledge of culture media's and consumables setting prior OPU or ET.

15 Excellent hands on skills of oocyte searching from follicular fluid and time management.

16 Excellent hands on skills of oocyte washing and conventional IVF with aseptic techniques.

\section{ABOUT EMBRYOLOGY LAB}

The Embryology laboratory is the place where all eggs are retrieved and fertilized with prepared semen sample; the embryos are further cultured and analyzed till day 5 by a team of embryologist. Also the embryos are vitrified and thawed in the same laboratory. Egg retrieval procedures are to be performed by infertility doctor in the operation theater located adjacent to the IVF Laboratory, which requires the highest level of ethical standards. To assure quality, each IVF laboratory requires accreditation and a license to operate issued by the ICMR as well as civil surgeon [10]. To run the ethical embryology lab following practices must be followed:

\section{Distinct Sterile and Non-sterile Areas [11]:}

There should be separate sterile and non-sterile areas in the In vitro fertilization lab. The sterile areas must include the operation theatre, embryology lab area where the embryos are incubated, andrology lab
Highly involvement in teaching and sharing new skills to other staffs.

Involvement in donor recruitment and selection of donor following ethical guideline.

Embryology and Andrology Lab SOP's need to be updated time to time by Embryologist.

Excellent hands on skills of TESA -ICSI, Frozen Sperm-ICSI and Frozen Oocyte - ICSI.

Excellent hands on skills of Frozen oocyte thawing and frozen oocyte - ICSI.

Excellent hands on skills of Physiological ICSI (PICSI) and management of Globozoospermia.

Superb techniques known to semen processing for IUI, IVF/ICSI or any other ART treatments.

Good knowledge of sperm function testing (semen analysis, sperm morphology, sperm DFI and SST).

which includes semen processing and IUI preparation and cryopreservation area. These areas should be fully air-conditioned with $100 \%$ power backup. Semi sterile areas include the general andrology lab for counseling the patient and receiving the semen sample, autoclave room, changing room. Non sterile zone are: store room, reception area and waiting room. Entry to the sterile areas of the lab should be restricted and only embryologists and IVF staffs in sterile clothes and footwear are to be allowed.

\section{Man Power / Staffing:}

According to Indian council of medical research (ICMR) the minimum requirement regarding staff in infertility clinics must require a gynaecologist, andrologist, clinical embryologist, counselor and a programme coordinator. A clinic that performs considerable number of IVF cycles annually should have 2 full-time embryologists on the team [12]. This enables doctors to retrieve eggs according to patient's 
convenience and not based on the availability of the embryologist.

\section{Quality Management:}

All equipment used in the lab should be serviced time to time. Any media or reagents being used should be tested regularly for any $\mathrm{pH}$ change. Every procedure performed in the lab should be witnessed by more than one staff which reduces the risk of human error. The embryologist must be responsible for checking quality management [11].

\section{Records:}

A good IVF lab should maintain proper records for all procedures performed. This includes procedure name, dates, time, staff involved, characteristics of embryos and any relevant communication with the patient. These records should be traceable and confidential [11].

\section{ABOUT ANDROLOGY LAB}

"Andrology" is the branch of medical science which deals with the study male reproduction as well as semen examination and processing. The Andrology Laboratory should provide therapeutic procedures for patients requiring assistance in achieving pregnancy such as Assisted Reproduction techniques: Intrauterine Insemination (IUI) and In Vitro Fertilization (IVF). The Andrology laboratory also works in parallel with endocrinology, genetic and in vitro fertilization laboratories to diagnose and assist in the treatment of patients facing difficulties in conceiving or seeking preservation of their reproductive capacity by cryopreserving their gametes or reproductive tissue. Semen analysis is the most common test to be conducted in the Andrology laboratory. Before working on design of the laboratory, regulations must be carefully studied. The regulations may differ from country to country or even between the states in the same country. Ideally, the Andrology laboratory should contain different areas for different procedures. Therapeutic procedures such as IUI or semen sample preparations should be performed in a sterile environment, which can be attained by using a laminar air flow hood. The area of lab should be determined according to the number of tests to be offered by the clinic and the total number of procedures expected to be performed. If the laboratory is going to offer cryopreservation, there should be space for the liquid nitrogen (LN2) containers that will store tissue samples. Due to the chemical properties of liquid nitrogen, a laboratory should follow the proper safety guidelines for area ventilation and oxygen monitoring. The semen collection room (SCR) is an important aspect of an Andrology laboratory. Therefore, it must be planned attentively to style, especially with regard to location. Usually the SCR is found in proximity to the laboratory. Places that provide privacy (avoid busy corridors, lobbies or reception areas) are appropriate.

Different work stations are used for performing different procedures, given below are the uses of those workstations:

Workstation 1: This area can be equipped with microscopes, cell counters, incubators, water baths or warm stage and also the necessary supplies and reagents. $37^{\circ} \mathrm{C}$ temperature is required for liquefaction, semen analysis (count and motility assessment), and HOS incubation. This workstation also can be equipped with Computer assisted semen analysis (CASA), which generates a more complete assessment of sperm motion characteristics.

Workstation 2: Staining and slide preparation for morphology. This bench is typically near a sink or a drain where the slides should be washed during staining. Staining solutions can be kept in plastic containers or glassware and contamination should be avoided. When morphological slides are ready, they should be transferred to morphology assessment bench. 
Workstation 3: Semen preparation and cryopreservation procedures. As mentioned previously, these procedures should be performed in an exceedingly sterile environment. When optimizing the semen preparation procedures, a $\mathrm{CO} 2$ incubator should be used for sample incubation. [13]

The safety guidelines for the Andrology laboratory include use of protective gloves and appropriate laboratory coats for all procedures. All samples must be handled very carefully as it may be potentially contaminated and harmful. The World Health Organization (WHO) laboratory manual in its fifth edition 2010 and six edition 2021, has described these guidelines [14].

\section{VII.DISCUSSION}

Clinical Embryologist actively involved in conventional in-vitro fertilization (IVF), and now in modern times there are many protocols that can be used to stimulate the ovaries of patients. The motive is to retrieve the optimal number of eggs required to get enough healthy embryos that ultimately lead to pregnancy. Short protocol/Antagonist protocol is the most ordinarily used IVF protocol. It involves less number of injections (which is also cost effective) and is very effective in results for the majority of patients. Patients could also be instructed to use contraceptive pills before starting an IVF cycle. For 10 days, folliclestimulating hormone (FSH) and luteinizing hormone (LH) injections are given. To prevent premature ovulation, the Gonadotropin-releasing Hormone $(\mathrm{GnRH})$ antagonist is added around fifth day. The doctor may use a "Lupron trigger" or "dual trigger" to stimulate the ultimate maturation of the oocytes before retrieval and significantly decrease the chance of Ovarian Hyperstimulation Syndrome (OHSS) [15]. With IVF, the trigger shot is employed before egg retrieval to help facilitate a process called meiosis. Ovulation generally occur about 36 to 40 hours after giving a trigger shot. Since the shot is employed differently in IUI and IVF, this suggests that the timing of the shot is vital in regard to the other procedures you're having. The doctor will monitor the ovaries via ultrasound and provides the green light to do the trigger shot when the follicles are reached between 16 and 18 millimetres. This is usually between days $8^{\text {th }}$ to $12^{\text {th }}$ of the cycle. After the shot is given, patient is scheduled for egg retrieval within 35-36 hours. Then the eggs are retrieved and further fertilized using the partner's or a donor's sperm. The fertilized eggs are then either transferred (when doing a fresh transfer) between 3 and 5 days after the retrieval or frozen (for later transfer). Since the trigger shot contains hCG, patient may be able to get a positive on a pregnancy test without being pregnant if they test too soon after the shot. Experts recommend waiting at least 2 weeks after the shot to take a pregnancy test, because it may take between 10 and 14 days for the trigger shot's effect to leave the system. And if doing ART procedures, the doctor may schedule you for a beta (initial) blood test to look for hCG [16]. If the test is positive, patients are advised for further pregnancy treatment. If the tests are negative, then patients are rescheduled for another cycle (FET).

\section{CONCLUSION}

An embryologist focuses on aseptic techniques and creating embryos outside the mother's womb with ICSI or the co-incubation of sperm and eggs. Through their expertise within the study of embryo cells, an embryologist focuses on discovering ways to treat reproductive and fertility issues. Above all, there's no better feeling than giving hope to the couple for parenthood who are unable to conceive on their own. This profession gives one a way of pride as you see an embryo turning into implantation and growing foetus. [17].

\section{CONFLICT OF INTEREST}

The authors declare no conflict of interest. 


\section{ACKNOWLEDGEMENTS}

We would like to thank the members of the study and guidance for writing about embryologist role in ART fields. We also thanks to the Director of IRCC Hospital \& IVF centre for their cooperation and motivational support.

\section{REFERENCES}

[1]. Embryology, Access online on 30/8/2021Embryology - Wikipedia].

[2]. Times of India, embryology: understanding the science and the scientist behind a successful IVF procedure access online on 30/8/2021, Embryology: Understanding the science and the scientists behind a successful IVF procedure (indiatimes.com)]

[3]. Scott L. Pronuclear scoring as a predictor of embryo development. Reprod Biomed Online 2003; 6: 201214.

[4]. Indian Fertility Society | Fertility Science \& Research. Diploma in Clinical Embryology | Indian Fertility Society.

[5]. ESHRE certification for clinical embryologists, Certification for clinical embryologists (eshre.eu).

[6]. Embryology Certificate Course, Embryology Certificate Course - Society of Reproductive Biologists Technologists (asrm.org).

[7]. Clinical Scientist, embryology job profile, access online on 10/09/2021 Clinical scientist, /embryology job profile | Prospects.ac.uk]

[8]. Clinical Embryology, Access online] on 25/08/2021, Clinical Embryology Course, Subjects, Syllabus, Colleges, Admission, Career (careers360.com)]

[9]. Hindustan Times, how to pursue a career in clinical embryology, Access online] on 25/08/20211, How to pursue a career in clinical embryology - Hindustan Times]

[10]. Fertility center of Illinois, what happens inside an embryology lab? Access online] on 24/08/2021, Sneak Peek: What Happens Inside an Embryology Laboratory? (fcionline.com)]

[11]. Nova IVF Fertility, what are the best practices of embryology lab management? Access online] on
25/08/2021, What are the Best Practices for Embryology Lab Management? NOVA IVF (novaivffertility.com)]

[12]. Introduction, Brief History of ART and Requirements of ART Clinics https://main.icmr.nic.in/sites/default/files/guidelin es/Chapter.pdf]

[13]. Kelly S Athayde, Ashok Agarwal, chapter no 16; Setting Up an Andrology Laboratory, download online on 27/08/2021 16.pmd (clevelandclinic.org)].

[14]. WHO laboratory manual for the Examination and processing of human semen, fifth edition 2010, untitled (who.int) and WHO laboratory manual for the examination and processing of human semen, sixth edition 2021 (WHO laboratory manual for the examination and processing of human semen).

[15]. Which IVF protocol is right for you?, access online on 08/09/2021 Which IVF Protocol is Right for You? (progyny.com)]

[16]. Trigger shot: what it is, how it works, side effects, more, access online 08/09/2021Trigger Shot: What It Is, how it Works, Side Effects, More (healthline.com)]

[17]. What is the role of embryologist in ivf treatment? Access online on 09/09/2021 What is the role of an Embryologist in IVF Treatment (pristyncare.com)]

[18]. Pronuclear Scoring in Human In Vitro Fertilization Pronuclear Scoring in Human In Vitro Fertilization | SpringerLink].

\section{Cite this article as:}

Harsimrat Kaur, Ram Dayal, Kamla Singh, "Embryologist's Job in Assisted Reproductive Technologies and in Vitro Fertilization", International Journal of Scientific Research in Science and Technology (IJSRST), Online ISSN : 2395602X, Print ISSN : 2395-6011, Volume 8 Issue 5, pp. 108114, September-October 2021. Available at doi: https://doi.org/10.32628/IJSRST218521 Journal URL : https://ijsrst.com/IJSRST218521 